\title{
Bronchoalveolar lavage cell analysis in a child with chronic lipid pneumonia
}

\author{
F. Midulla*, P.M. Strappini**, V. Ascoli+, M.P. Villa*, L. Indinnimeo*, \\ C. Falasca*, S. Martella*, R. Ronchetti*
}

Bronchoalveolar lavage cell analysis in a child with chronic lipid pneumonia. F. Midulla, P.M. Strappini, V. Ascoli, M.P. Villa, L. Indinnimeo, C. Falasca, S. Martella, R. Ronchetti. CERS Journals Ltd 1998.

ABSTRACT: In an asymptomatic $4 \mathrm{yr}$ old child with radiographic evidence of parenchymal lung disease, bronchoalveolar lavage (BAL) yielded the diagnosis of chronic lipid pneumonia caused by chronic aspiration of mineral oil given as a laxative. BAL analysis showed a marked reduction in the total number of alveolar macrophages; almost $\mathbf{7 0 \%}$ of these cells contained intracytoplasmic lipid vacuoles. It also disclosed lymphocytic (cytotoxic/suppressor) alveolitis. A high percentage of lymphocytes expressed antigen markers of activation (human leucocyte antigen (HLA)-DR), CD54 and CD25). BAL analysis 18 months after mineral oil intake revealed that lymphocytes bearing antigen markers of activation had markedly decreased whereas alveolar macrophages (normal and lipid-laden) had increased. A subsequent whole lung BAL was considered unnecessarily invasive in this otherwise healthy child.

Eur Respir J 1998; 11: 239-242.
*IV Dept of Pediatrics, Institute of Pediatrics, **Biochemistry Laboratory, Institute of Pediatrics, and +Dept of Experimental Medicine and Pathology, University of Rome "La Sapienza", Italy.

Correspondence: F. Midulla

IV Cattedra di Clinica Pediatrica

Istituto di Clinica Pediatrica

Università di Roma "La Sapienza"

Viale Regina Elena

324. 00161 Rome

Italy

Fax: 003964462767

Keywords: Bronchoalveolar lavage, cellular components, children, lipid pneumonia Received: February 121997

Accepted after revision August 21997
Lipid pneumonia (LP) is rarely responsible for chronic parenchymal lung disease in children [1]. It is most commonly caused by the aspiration of milk [2], mineral oil [3], oily nose drops [4] and lip gloss [5]. Possible predisposing factors include certain traditions, such as forcible administration of rendered animal fat to neonates and infants [6].

The diagnosis of LP depends partly on the demonstration of lipid-laden macrophages in bronchoalveolar lavage (BAL) fluid [7]. LP represents a foreign body reaction to the oil. Its clinical presentations vary widely. In some cases it is an incidental finding in an asymptomatic patient; in others it causes acute respiratory distress [8]. The sequence of immunological responses that may culminate in chronic alveolar damage are poorly understood.

We report the BAL cytological findings in a child with LP caused by chronic aspiration of liquid paraffin administered as a laxative. To our knowledge this is the first paediatric report describing BAL cell differentials and lymphocyte subsets at the time of diagnosis, and at 6, 12 and 18 months after mineral oil intake had been discontinued.

\section{Case report}

A 4 yr old boy was admitted to our Department after radiographic evidence displayed consolidation in the left lower lobe and multiple patchy infiltrates in the right lung (fig. 1a). These findings were later confirmed by computed tomography (CT) scan (fig. 1b). The patient was a healthy Caucasian Romanian boy who had been adopted at the age of 3 months. Since the age of 4 months he had suffered from chronic constipation.

On admission, the patient appeared physically healthy. Pulse oximetry yielded $98 \% \mathrm{O}_{2}$ saturation (room air). The white blood cell counts were $6.4 \times 10^{9}$ cells $\cdot \mathrm{L}^{-1}$, neutrophils 53\%, lymphocytes 37\% (CD3 67\%, CD19 21\%, CD3CD4 29\%, CD3CD8 27\%, CD4/CD8 1.1, CD3DR 7\% CD3CD25 0\%, CD3CD54 0\%), monocytes 6\% and eosinophils $4 \%$. Sedimentation rate was $5 \mathrm{~mm} \cdot \mathrm{h}^{-1}$. Serum cholesterol was $216 \mathrm{mg} \cdot \mathrm{dL}^{-1}$ and triglycerides were 81 $\mathrm{mg} \cdot \mathrm{dL}^{-1}$. Routine blood chemistry was normal. Delayed hypersensitivity skin tests demonstrated positive reactions to diphtheria and tetanus. A tuberculin skin test was negative. Skin-prick tests were positive for grass pollen. A technetium-99m isotope lung scan showed perfusion defects in the left lower lobe and in the right upper lobe. The child underwent fibreoptic bronchoscopy (FB) and BAL after informed parental consent. After topical lidocaine anaesthesia of the upper airways and sedation of the child with meperidine $1-2 \mathrm{mg} \cdot \mathrm{kg}^{-1} i . v$, FB was performed using a Pentax FB $10 \mathrm{H}$ fibreoptic bronchoscope (Tokyo, Japan). During FB the child was breathing spontaneously, and supplemental $\mathrm{O}_{2}$ was administered through a nasal catheter. The patient was monitored during the procedure for cardiac frequency and $\mathrm{O}_{2}$ saturation. BAL fluid was obtained by instilling three $10 \mathrm{~mL}$ aliquots of prewarmed sterile saline via the suction channel of the bronchoscope into the left lower lobe. Each aliquot was immediately suctioned back into the same syringe and subsequently stored in ice. The first aliquot was used for microbiological studies, the second and third aliquots were pooled together and used 
a)

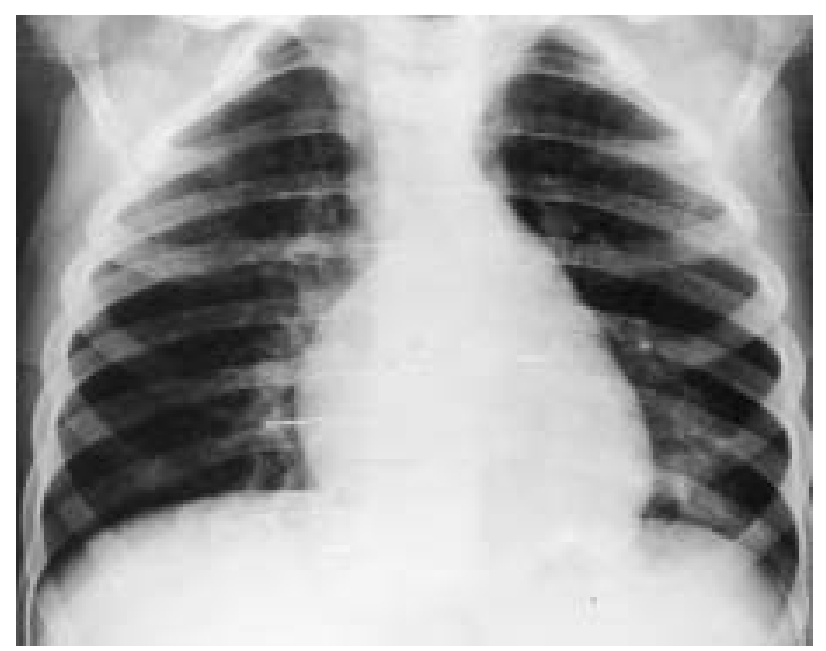

b)

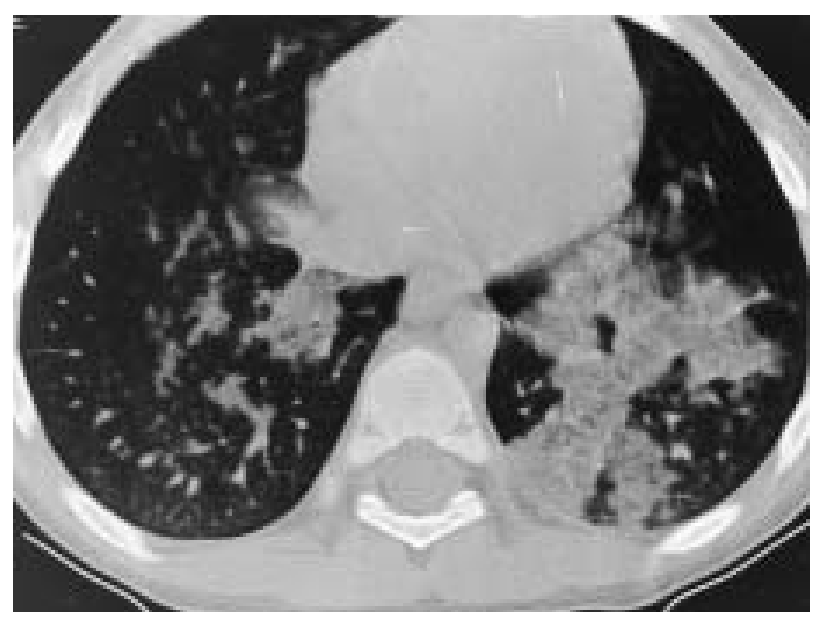

Fig. 1. - a) Chest radiograph showing diffuse parenchymal consolidation of the left lower lobe and patchy infiltrates in the right upper lobe. b) Computed tomography scan showing bilateral alveolar consolidation, interlobular septal thickening and lymphatic infiltration.

for cellular analysis. Differential cell counts were determined on May-Grunwald-Giemsa stained cytospin preparations $\left(10^{5}\right.$ cells $\left.\cdot 0.5 \mathrm{~mL}^{-1}\right)$ from at least 200 cells [9]. FB examination showed a normal bronchial tree. Direct microscopic observation of BAL fluid cytospin preparations revealed a marked reduction of alveolar macrophages and a markedly increased number of lymphocytes as compared to normal values $[9,10]$ (table 1). About $70 \%$ of the macrophages had multiple round intracytoplasmic vacuoles (fig. 2a). Oil Red O staining demonstrated that these inclusions contained lipids (fig. 2b). Electron microscopic evaluation of BAL cells confirmed the presence of intracytoplasmic vacuoles varying in size and filled with lipid-like material (fig. 2c). Flow cytometry analysis (FACScan; Becton-Dickinson, San Jose, CA, USA) of BAL lymphocytes revealed a cytotoxic/ suppressor pattern of lymphocytic alveolitis. A high percentage of CD3 lymphocytes expressed human leucocyte antigen-D region (HLA-DR) (class II major histocompatibility complex molecule), CD25 (interleukin (IL)-2 receptor) and CD54 (intracellular adhesion molecule, (ICAM)-1).
Table 1. - Results of bronchoalveolar lavage (BAL) during mineral oil aspiration, and 6,12 and 18 months after the discontinuation of oil intake

\begin{tabular}{|c|c|c|c|c|c|}
\hline & \multicolumn{4}{|c|}{$\begin{array}{l}\text { Left lower lobe } \\
\text { Follow-up months }\end{array}$} & \multirow{2}{*}{$\begin{array}{c}\text { Normal } \\
\text { reference } \\
\text { values } \\
{[9,10]}\end{array}$} \\
\hline & 0 & 6 & 12 & 18 & \\
\hline BAL recovery & 62 & 65 & 85 & 57 & $43 \pm 3$ \\
\hline Cells $\cdot \mathrm{mL}^{-1} \times 10^{3}$ & 600 & 500 & 700 & 900 & $600 \pm 82$ \\
\hline \multicolumn{6}{|c|}{ Differential cell counts } \\
\hline $\mathrm{AM} \%$ & 26 & 67 & 67 & 57 & $86 \pm 2$ \\
\hline$\times 10^{3} \cdot \mathrm{mL}^{-1}$ & 156 & 335 & 469 & 513 & $500 \pm 64$ \\
\hline Lymphocytes \% & 68 & 32 & 23 & 38 & $9 \pm 1$ \\
\hline$\times 10^{3} \cdot \mathrm{mL}^{-1}$ & 408 & 160 & 161 & 342 & $60 \pm 6$ \\
\hline Neutrophils \% & 2 & 0 & 8 & 5 & $6 \pm 1$ \\
\hline$\times 10^{3} \cdot \mathrm{mL}^{-1}$ & 12 & 0 & 56 & 45 & $39 \pm 13$ \\
\hline Eosinophils \% & 4 & 1 & 2 & 0 & $0.2 \pm 0.1$ \\
\hline$\times 10^{3} \cdot \mathrm{mL}^{-1}$ & 24 & 5 & 14 & 0 & $0.5 \pm 0.4$ \\
\hline \multicolumn{6}{|c|}{ Lymphocyte subsets \% of lymphocytes } \\
\hline T-lymphocytes CD3 & 90 & 86 & 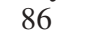 & 87 & $86(72-92)$ \\
\hline B-lymphocytes CD19 & 2 & 5 & 4 & - & $1(0-7)$ \\
\hline $\begin{array}{l}\text { Helper/Inducer CD4 } \\
\text { Suppressor/ }\end{array}$ & 32 & 35 & 28 & 36 & $33(10-57)$ \\
\hline Cytotoxic CD8 & 67 & 50 & 53 & 48 & $57(30-84)$ \\
\hline $\mathrm{CD} 4 / \mathrm{CD} 8$ ratio & 0.5 & 0.7 & 0.5 & 0.7 & $0.7(0.1-1.9)$ \\
\hline \multicolumn{6}{|l|}{ Class II MHC- } \\
\hline molecule CD3DR & 61 & 26 & 8 & 9 & - \\
\hline ICAM-1 CD3CD54 & 20 & 31 & 9 & - & - \\
\hline \multicolumn{6}{|l|}{ Interleukin (IL)-2 } \\
\hline receptor $\mathrm{CD} 3 \mathrm{CD} 25$ & 7 & 5 & 2 & 2 & - \\
\hline
\end{tabular}

Values are presented as mean $\pm S D$ with range in parenthesis. ICAM-1: intracellular adhesion molecule-1. AM: alveolar macrophages; MHC: major histocompatibity complex.

Upon further questioning, the mother revealed that for the past 2 yrs the child had been treated with oral paraffin oil for constipation. She described having difficulty with administration. The child refused the medication vigorously, often gagging and coughing during ingestion. The administration of paraffin oil was suspended and the child was discharged from the hospital.

Owing to the highly abnormal BAL results, showing a marked reduction of alveolar macrophages, the presence of lipid-laden cells and a high number of activated T-lymphocytes, we seriously considered long-term corticosteroid treatment. Given the child's good clinical conditions, however, we decided only to stop paraffin oil administration and to reconsider steroid treatment after a second BAL examination. A second BAL examination performed 6 months later, when the clinical situation remained normal, revealed an improvement in BAL results with a reduced absolute number of T-lymphocytes, especially of activated cells (table 1 and fig. $3 b)$. But the absolute number of alveolar macrophages remained low (about half of the expected normal values) and the number of lipid-laden cells had even increased (table 1 and fig. 3a). These contrasting findings and the good clinical conditions of the patient prompted us to use BAL again for clinical follow-up, without starting medical treatment. The third and fourth BAL procedures, performed 12 and 18 months after the diagnosis, showed that activated T-lymphocytes had almost completely disappeared and the total number of alveolar macrophages had normalized, though half of them were 
a)

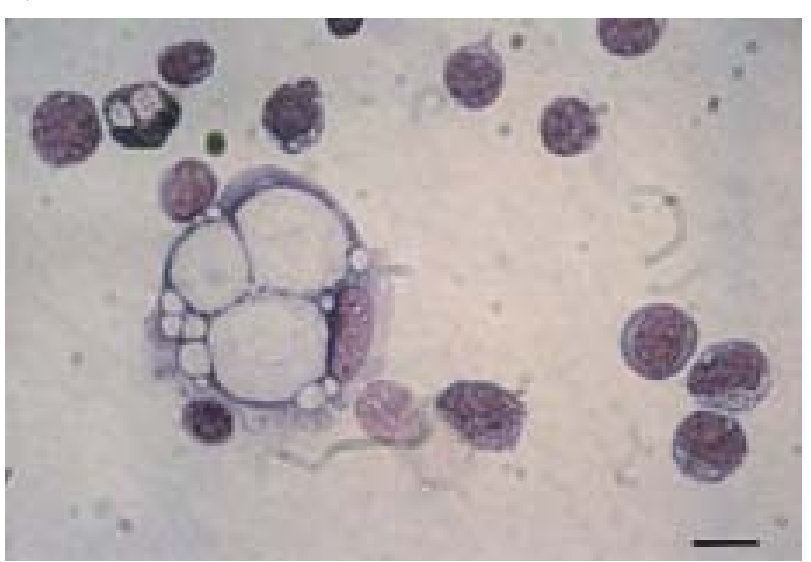

b)

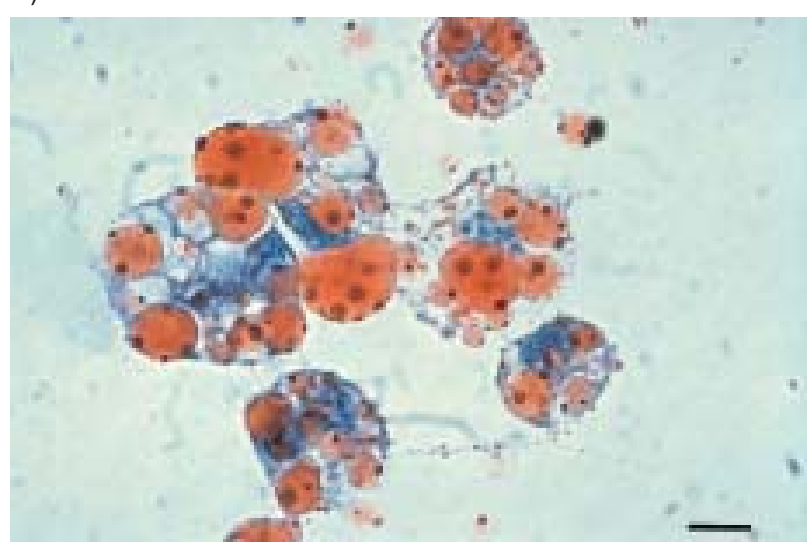

c)

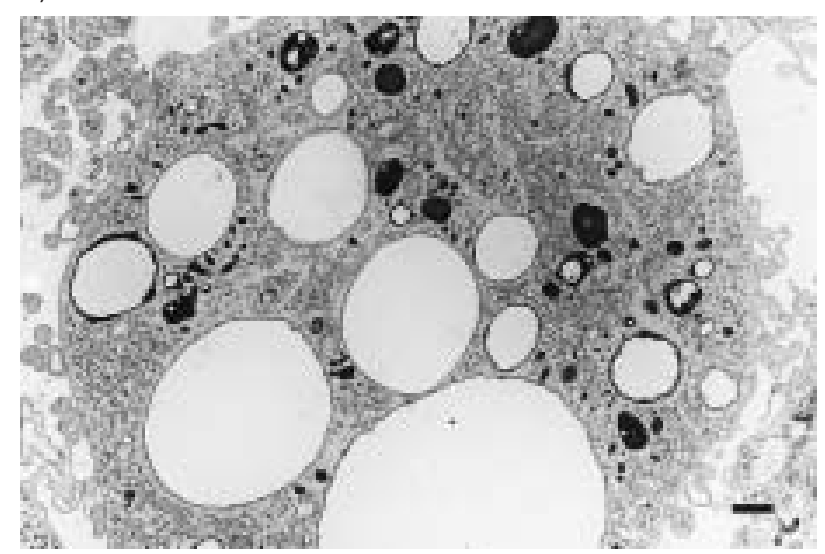

Fig. 2. - a) Alveolar macrophage with a small, bland nucleus and several intracytoplasmic empty-looking vacuoles. (Internal scale bar = $10 \mu \mathrm{m})$. b) Oil Red $\mathrm{O}$ staining demonstrating fat in the cytoplasm. (Internal scale bar=10 $\mu \mathrm{m}$ ). c) Intracytoplasmic vacuoles of various sizes. Larger spaces appear electronlucent, while smaller vacuoles are filled with highly electrodense (i.e. osmiophilic) material. Note also the medium-sized inclusions bounded by an osmiophilic "ring". This electrodensity provides evidence that the intracytoplasmic vacuoles contained lipid-like material. (Internal scale bar $=0.3 \mu \mathrm{m}$ ).

still lipid-laden (figs. 3a and b). There were fewer lipidladen macrophages in the last of the six BAL aliquots than in the first (fig. 3c). A chest radiograph obtained $1 \mathrm{yr}$ after the diagnosis demonstrated a marked reduction in the lung consolidation, whereas the perfusion scan abnormalities remained unchanged, suggesting persisting parenchymal damage.
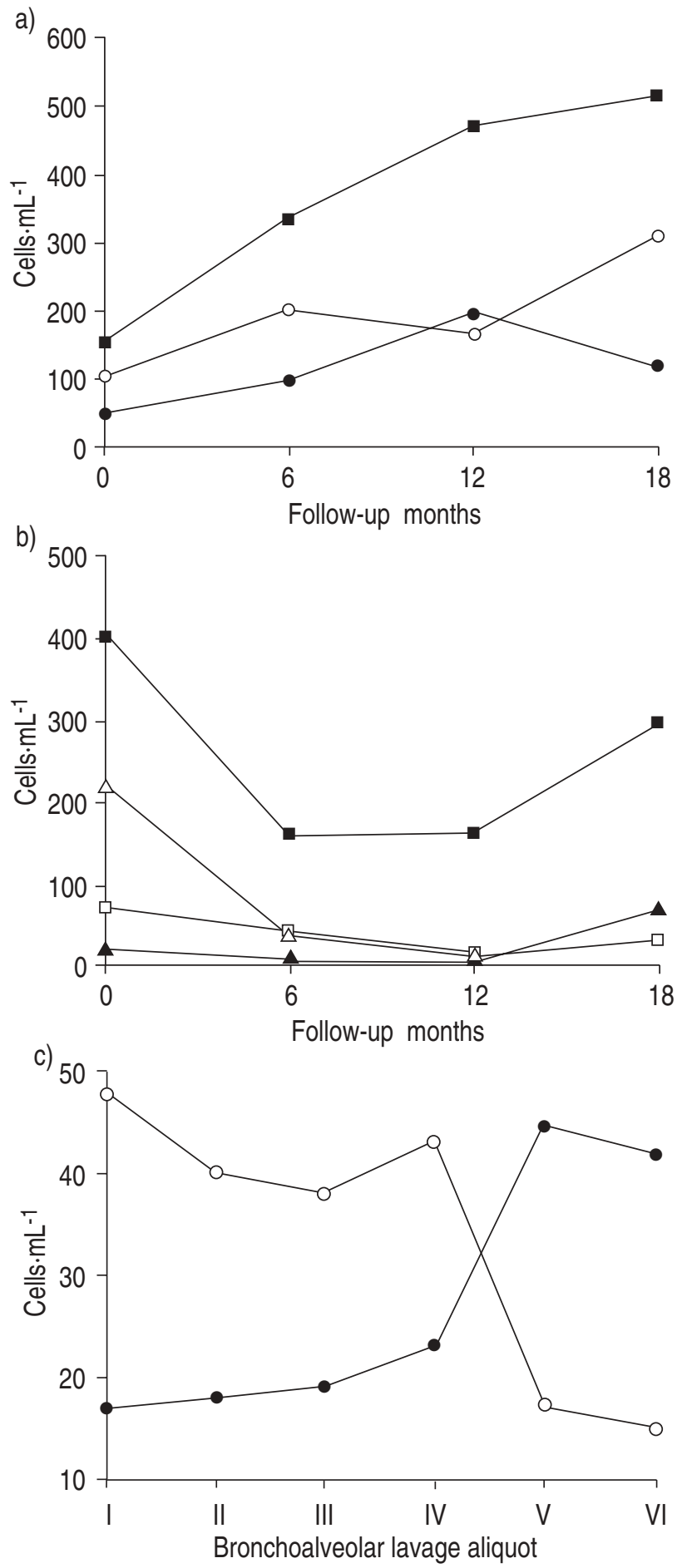

Fig. 3. - a) Lipid-laden and normal alveolar macrophages in bronchoalveolar lavage (BAL) fluids during 18 months of follow-up. $\mathbf{\square}$ : total; •: normal; ○: lipid-laden. b) Lymphocytes and lymphocytes bearing surface antigen markers of activation in BAL fluids. $\mathbf{~ : ~ l y m - ~}$ phocytes; $\Delta$ : CD3DR; $\square:$ CD3CD54; $\Delta$ : CD3CD25. c) The percentage of lipid-laden macrophages progressively decreased during sequential BAL analysis. Note that six aliquots of $10 \mathrm{~mL}$ each were used for the last BAL examination (at 18 months of follow-up). $\bullet$ : normal; $\bigcirc$ : lipid-laden.

\section{Discussion}

The clinical diagnosis of chronic LP is complex. One reason is the difficulty in ascertaining the history of oil ingestion. In addition, the symptoms appear only at an 
advanced stage of the illness and its biological, radiological and functional features are not disease specific [8]. Although several tools have been proposed for the diagnosis of LP $[6,11]$, BAL demonstrating lipid-laden macrophages remains the gold standard [7, 12].

BECTON et al. [5] have reported that almost $50 \%$ of patients with LP are asymptomatic. In many cases the disease is discovered by chance, during a routine chest radiograph. The clinical symptoms (chest pain, dyspnoea, cough and fever) vary according to the duration of oil intake, and the amount and quality of oil aspirated. Mineral oil aspiration may initially be clinically inapparent, stimulating neither the gag reflex nor coughing.

Although the precise mechanisms underlying oil-induced damage to the lung remain unclear, it has been suggested that aspirated oil is emulsified and phagocytosed by alveolar macrophages, which are then damaged. The lipids are released again locally, inciting chronic inflammation eventually leading to fibrosis [12].

During the clinical follow-up of a patient with chronic LP induced by mineral oil aspiration, we analysed BAL differential cell counts and lymphocyte surface antigens, during mineral oil intake and after administration had been discontinued. In this report we have used these results retrospectively to investigate the inflammatory response induced by mineral oil aspiration in the lung. The most striking BAL findings in our patient during oil aspiration were the prominent lipid-laden macrophages, the marked reduction in normal alveolar macrophages, the slight increase in eosinophil numbers and the increased number of activated lymphocytes. After oil intake ended, normal and lipid-laden macrophages increased and lymphocyte numbers decreased (table 1 and figs. $3 a$ and $b$ ). The presence of lipid-laden macrophages confirms that the oil is phagocytosed by alveolar macrophages. The low number of alveolar macrophages in the first BAL fluid sample (obtained during oil aspiration) suggests that these cells died because they were engulfed with oily material. BAL analysis 18 months later showed that alveolar macrophage numbers had returned to normal. The persistence of lipid-laden macrophages confirmed that clearance of aspirated oil from the respiratory tract is a slow process, possibly pathogenically contributing to the fibrosis eventually seen in this disease [12].

Although lymphocyte numbers sharply decreased after mineral oil intake ended; lymphocytic alveolitis persisted even after 18 months of follow-up. Lymphocyte subset analysis in BAL samples showed a predominance of CD3CD8 positive cells. In the first BAL fluid sample many CD3 lymphocytes expressed antigen surface markers of activation (DR, CD54 and CD25) suggesting a $\mathrm{T}$ cell-class II major histocompatibility complex (MHC) restricted response. Sequential BAL analysis showed a progressive decrease in the percentage of CD3DR, CD3CD54 and CD3CD25 positive lymphocytes, demonstrating that the lymphocyte population remaining in the alveolar spaces were in a less activated state. This is not a surprising finding because lymphocytic alveolitis, a typical feature of several lung diseases, can last for several years after clinical recovery [13-15].

Corticosteroid therapy was performed in all symptomatic paediatric patients with LP reported by ANNOBIL et al. [6]. Treatment in patients without clinical symp- toms remains controversial. In this child we noted fewer lipid-laden macrophages in the last BAL aliquot (when six aliquots were used) than in the first (fig. 3c). This observation suggests that whole lung lavage is the only method capable of removing the oil from the lung. SACCO et al. [16] recently described a case of mineral oil aspiration in a patient with acute exacerbation of dyspnoea successfully treated by whole lung lavage.

Since only a single case was observed here, we cannot determine the optimal algorithm for treatment of those asymptomatic case. However, close monitoring of the disease progression is advisable. The decision on whole lung bronchoalveolar lavage should be based on the clinical severity of the case.

\section{References}

1. Fan LL, Langston C. Chronic interstitial lung disease in children. Pediatr Pulmonol 1993; 16: 184-196.

2. Moran TS. Milk aspiration pneumonia in human and animal subjects. Arch Pathol Lab Med 1953; 55: 286-301.

3. Fan LL, Graham LM. Radiological cases of the month. Arch Pediatr Adolesc Med 1994; 148: 205-206.

4. Lipinski JK, Weisbrod GL, Sander DE. Exogenous lipid pneumonitis. J Can Assoc Radiol 1980; 31: 92-94.

5. Becton DL, Lowe JE, Falletta JM. Lipid pneumonia in an adolescent girl secondary to use of lip gloss. J Pediatr 1984; 105: 421-425.

6. Annobil SH, Ogunbiyi AO, Benjamin B. Chest radiographic findings in childhood lipoid pneumonia following aspiration of animal fat. Eur J Radiol 1993; 16: 217-220.

7. Spatafora M, Bellia V, Ferrara G, Genova G. Diagnosis of a case of lipid pneumonia by bronchoalveolar lavage. Respiration 1987; 52: 154-156.

8. Gondouin A, Manzoni Ph, Ranfaing E, et al. Exogenous lipid pneumonia: a retrospective multicentre study of 44 cases in France. Eur Respir J 1996; 9: 1463-1469.

9. Midulla F, Villani A, Merolla R, Bjermer L, Sandstrom T, Ronchetti R. Bronchoalveolar lavage studies in children without parenchymal lung disease: cellular constituents and protein level. Pediatr Pneumol 1995; 20: 112-118.

10. Ratjen F, Bredendick M, Zheng L, Brendel M, Costabel U. Lymphocyte subsets in bronchoalveolar fluid of children without bronchopulmonary disease. Am J Respir Crit Care Med 1995; 152: 174-178.

11. Joshir R, Cholankeril JV. Computed tomography in lipoid pneumonia. J Comput Assist Tomogr 1985; 9: 211-213.

12. Lauque D, Dongay G, Levade T, Caratero C, Carles P. Bronchoalveolar lavage in liquid paraffin pneumonitis. Chest 1990; 98: 1149-1155.

13. Drent M, Van Velzen BH, Diamant M, Wagenaar SJSC, Hoogsteden HC, Van Den Bosch JMM. Bronchoalveolar lavage in extrinsic allergic alveolitis: effect of time elapsed since antigen exposure. Eur Respir J 1993; 6: 1276-1281.

14. Winterbauer RH, Lammert J, Selland M, Wu R, Corley D, Springmeyer SC. Bronchoalveolar lavage cell populations in the diagnosis of sarcoidosis. Chest 1993; 104: 352-361.

15. Myou S, Fujimura M, Yasui M, Ueno T, Matsuda T. Bronchoalveolar lavage cell analysis in measles viral pneumonia. Eur Respir J 1993; 6: 1437-1442.

16. Sacco O, Moroni C, Ciravegna B, et al. Lipoid pneumonia in a child with anoxic encephalopathy: treatment by whole lung lavage. Eur Respir J 1996; 9(23): 105S. 